Submission ID: 43835

\title{
The Approach to Processing the Data of Induction Logging in Horizontal
} Wells in the Presence of the Fault

M.G. Persova* (Novosibirsk State Technical University), Y.G. Soloveichik (Novosibirsk State Technical University), Y.I. Koshkina (Novosibirsk State Technical University), D.V. Vagin (Novosibirsk State Technical University), D.S. Kiselev (Novosibirsk State Technical University), I.I. Patrushev (Novosibirsk State Technical University)

\section{SUMMARY}

This paper proposes the approach to data processing, which allows quite a fast determination of the fault location using the induction logging data obtained in horizontal wells. The proposed approach is based on the use of the 1-D inversion and inversion based on the use of 2.5-D or 3-D finite element modeling. At the first stage, we determine a zone which is characterized by the dramatic change of the geoelectrical model parameters obtained as a result of the 1-D inversion in the adjacent points on the logging tool motion trajectory. Then, in this zone, we perform the multidimensional inversion which results in determining the point of intersection of the fault line and trajectory of the tool motion. The possibilities of the proposed approach are shown with the use of synthetic data obtained by 2.5-D modeling for the geoelectrical model which is specific for the problems of induction logging in horizontal wells. We show that the location of the point of intersection of the fault line and trajectory of the tool motion is determined quite accurately, and the computational time, which is required for the multidimensional processing in a separate zone, is about a minute when we use a personal computer. 
Подход к обработке данных индукционного каротажа в горизонтальных скважинах в присутствии разлома

М.Г. Персова* (НГТУ), Ю.Г. Соловейчик (НГТУ), Ю.И. Кошкина (НГТУ), Д.В. Вагин (НГТУ), Д.С. Киселев (НГТУ), И.И. Патрушев (НГТУ)

\section{Введение}

На сегодняшний день обработка данных индукционного каротажа (Davydycheva 2010, Liang et al. 2011), основанного на частотном электромагнитном зондировании околоскважинного пространства при бурении горизонтальных скважин, осуществляется в рамках слоистых моделей - с использованием одномерных подходов. Такая обработка, в целом, дает достаточно адекватные результаты при отсутствии в изучаемой среде резких изменений - разломов, влекущих за собой вертикальный сдвиг слоев.

В терминах рассматриваемых задач, эта ситуация приводит к тому, что геометрия среды становится, как минимум, существенно двумерной. Это, в свою очередь, требует использования многомерных подходов к обработке данных вместо одномерных. При этом применение многомерных подходов, с одной стороны, должно приводить к возможности получения адекватных геологических моделей, а с другой, не переводить обработку в разряд вычислительно трудоемких задач, чтобы оставалась возможность ее использования в производственном цикле.

В данной работе будет предложен подход, сочетающий одномерную и многомерную обработку данных индукционного каротажа в горизонтальных скважинах и позволяющий достаточно быстро определить местоположение разлома.

\section{Подход к определению местоположения разлома по данным индукционного каротажа в горизонтальных скважинах}

Предлагаемый подход к обработке данных индукционного каротажа в горизонтальных скважинах основан на использовании $1 \mathrm{D}$-инверсии и инверсии, основанной на использовании $2.5 \mathrm{D}$ или $3 \mathrm{D}$ конечноэлементного моделирования. При этом он состоит из нескольких этапов. На первом этапе на текущем участке скважины выполняется 1D-инверсия. По ее результатам определяется участок резкого изменения параметров среды. На этом участке выполняется инверсия, основанная на многомерном моделировании. Ее методика представлена в следующем разделе на примере.

Электромагнитное поле в процедуре многомерного моделирования ищется с использованием технологии разделения поля на нормальную $\overrightarrow{\mathbf{E}}^{n}$ и аномальную $\overrightarrow{\mathbf{E}}^{a}$ составляющие. Нормальная составляющая описывает поле источника в горизонтально-слоистой вмещающей среде и ищется с использованием подхода, представленного в (Persova et al. 2016). Аномальная составляющая ищется путем конечноэлементного решения уравнения

$$
\frac{1}{\mu_{0}} \nabla \times \nabla \times \overrightarrow{\mathbf{E}}^{a}+i \omega \sigma \overrightarrow{\mathbf{E}}^{a}=-i \omega\left(\sigma-\sigma^{n}\right) \overrightarrow{\mathbf{E}}^{n},
$$

где $\mu_{0}$ - магнитная проницаемость вакуума, $\sigma$ - тензор проводимости в 2D или 3D среде, $\sigma^{n}$ - тензор проводимости вмещающей среды, $\omega$ - круговая частота, $i$ - мнимая единица.

Подход к многомерной нелинейной инверсии с поиском координат границ структурных частей геоэлектрической модели представлен в работах (Persova et al. 2013, Mogilatov et al. 2016). 


\section{Пример}

В качестве примера применения предлагаемого подхода рассмотрим типичную для задач индукционного каротажа геоэлектрическую модель с разломом, представленную на рис. 1. Электромагнитное поле возбуждается индукционной катушкой, ось которой совпадает с направлением траектории, на двух частотах 40 кГц и 100 кГц, а принимается двумя приемниками с разносами 8 м и 12 м. Каротажный прибор фиксирует отношения амплитуд и разность фаз ЭДС в приемниках.

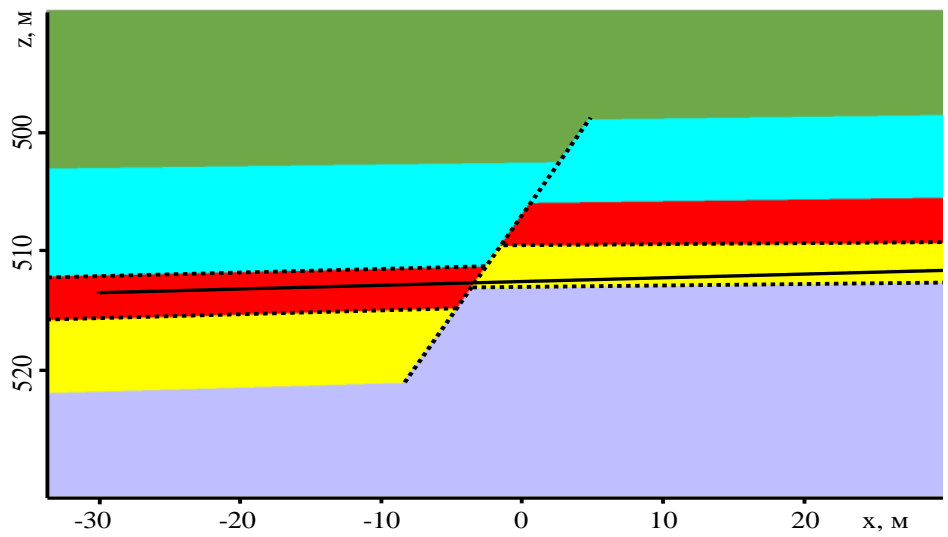

Рисунок 1 Истинная геоэлектрическая модель с разломом. Сплошной черной линией показана траектория движения каротажного прибора. Черными точками показано положение ближайших к траектории грании

Сначала проанализируем, каков размер зоны около разлома, внутри которой измеряемые сигналы не соответствуют одномерной (слоистой) модели, построенной по истинной среде в точке, соответствующей «нулю» каротажного прибора. Графики разности фаз и отношений амплитуд, рассчитанные для модели, представленной на рис. 1, в сравнении с графиками, рассчитанными для 1D-моделей, которые в каждой точке аппроксимируют рассматриваемую среду, представлены на рис. 2. Видно, что принципиальное отличие истинных и 1D-кривых наблюдается только в области внутри выделенного прямоугольника - далее будем называть эту зону участком «неодномерности». На практике этот участок определяется резким изменением в соседних точках параметров среды, подобранной с использованием 1Dинверсии.

(a)

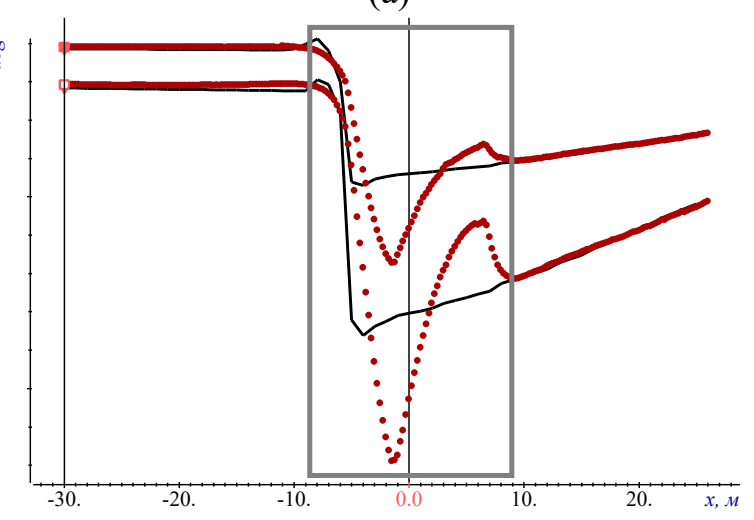

(б)

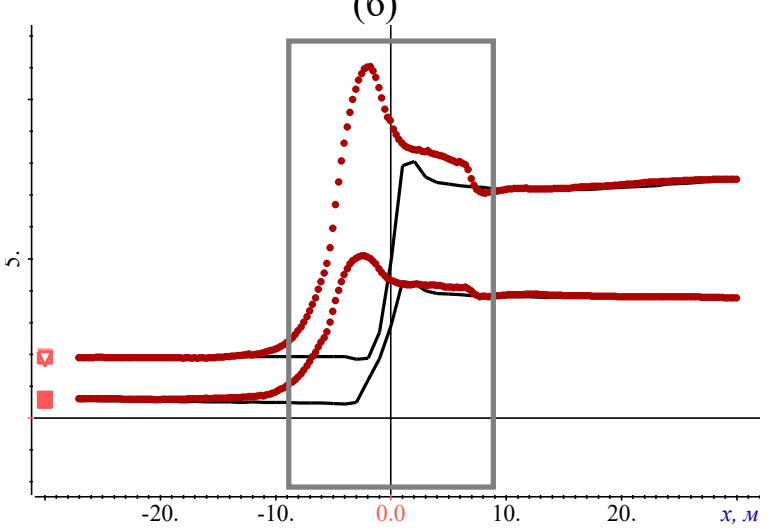

Рисунок 2 Графики разности фаз (а) и отношения амплитуд (б), рассчитанные для истинной модели (красные точки) и для $1 D$-моделей (черный цвет) для двух частот 40 и $100 \kappa Г и$

Выполнять многомерную инверсию для участка «неодномерности» можно в два этапа. Стартовая модель (рис. За) для первого этапа инверсии формируется следующим образом. До и после выделенного участка геоэлектрическая модель строится на основе результатов 1D- 


\section{EAGE}

инверсии. В центре выделенного участка «неодномерности» задается стартовое положение «линии разлома». Затем в левой и в правой части от выделенной линии внутри участка «неодномерности» задается два «столбика» объектов, при этом количество, мощность и положение объектов в столбиках определяется результатом 1D-инверсии в ближайших точках слева и справа участка «неодномерности». Таким образом, на этом этапе подбирается один параметр - положение «линии разлома».

Полученная в результате выполнения этого этапа модель показана на рис. 3б. Видно, что положение точки пересечения линии разлома с траекторией перемещения прибора была найдена практически точно, при этом значение функционала невязки уменьшилось в 4.5 раза. Время счета для этого этапа составило около 1 мин с использованием четырех ядер процессора Intel I7.

(a)

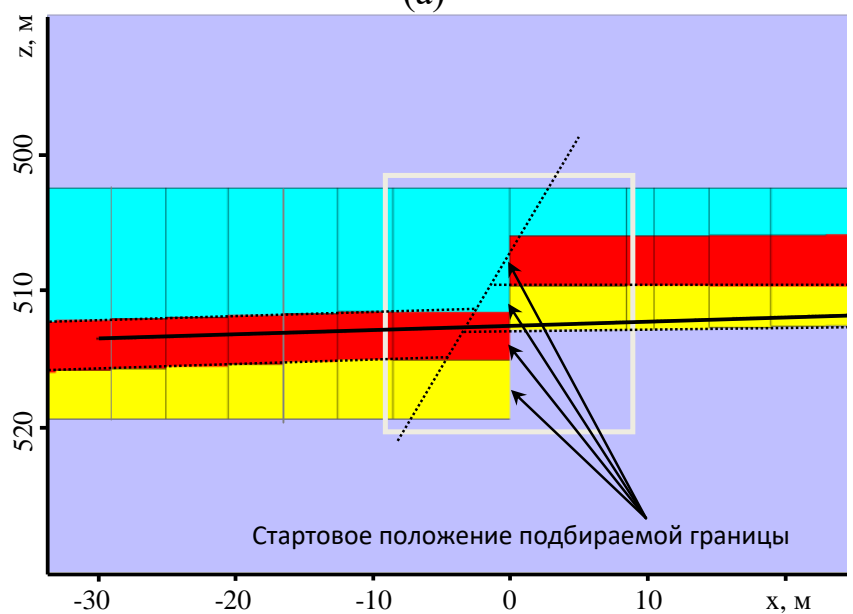

(б)

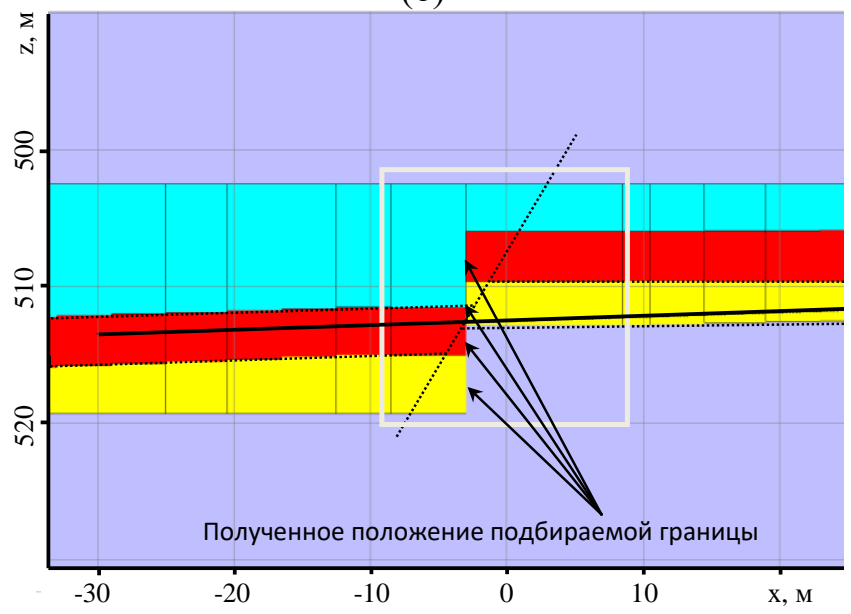

Рисунок 3 Стартовая (а) и подобранная (б) модели (черными точками показано истинное положение ближайших к траектории грании слоев и границы разлома)

В принципе, на этом этапе обработка участка «неодномерности» может быть прекращена. Однако, проанализировав показанные на рис. 4a,6 графики разности фаз для стартовой и подобранной моделей в сравнении с истинными, можно отметить, что, возможно, требуется второй этап инверсии и некоторое уточнение модели в окрестности разлома. Для этого в качестве искомых параметров были взяты участки ближайших к траектории горизонтальных границ слоев, расположенные внутри участка «неодномерности». Графики, полученные для модели, подобранной на втором этапе, показаны на рис. 4в. Видно, что они уже очень близки к истинным, однако сама модель изменилась очень незначительно. Поэтому этот этап инверсии не является обязательным и на практике вполне можно ограничиться только первым этапом. 
(a)

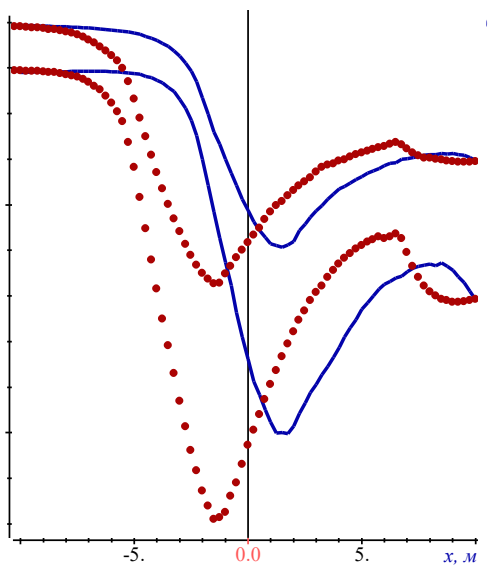

(б)

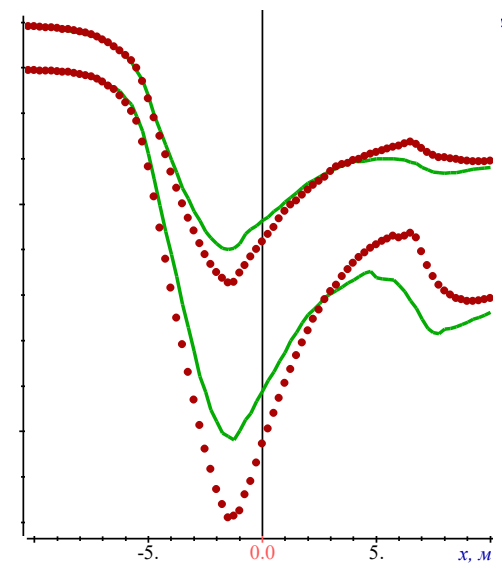

(в)

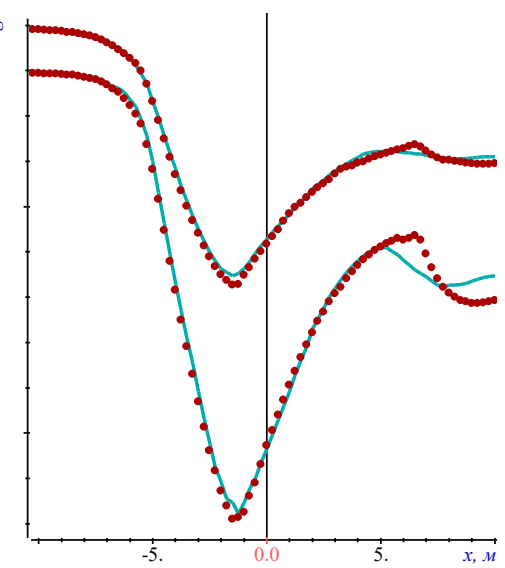

Рисунок 4 Графики разности фаз для стартовой модели (а) и моделей, подобранных после первого (б) и второго (в) этапов многомерной инверсии (красными точками показаны кривые для истинной модели, сплошные линии - для стартовой и подобранных моделей)

\section{Выводы}

Предложенный подход позволяет на основе анализа результатов 1D инверсии данных индукционного каротажа в горизонтальной скважине выделять участки «неодномерности», соответствующие зоне разлома, и с использованием многомерной инверсии и конечноэлементного 2.5D моделирования определять положение точки пересечения «линии разлома» и траектории перемещения прибора. При этом время, требуемое на выполнение многомерной обработки на выделенном участке, составляет порядка минуты на персональном компьютере.

\section{Благодарности}

Работа выполнена при финансовой поддержке Министерства образования и науки РФ (гос. задание, проект № 5.978.2017/4.6) и гранта Президента Российской Федерации для государственной поддержки молодых российских ученых - докторов наук (№ гранта МД-3516.2017.5).

\section{References}

Davydycheva S. [2010] 3D modeling of new-generation (1999-2010) resistivity logging tools. The Leading Edge, 29(7), 780-789.

Liang L., Abubakar A., and Habashy T. [2011] Estimating petrophysical parameters and average mud-filtrate invasion rates using joint inversion of induction logging and pressure transient data. Geophysics, 76(2), E21-E34.

Mogilatov, V., Goldman, M., Persova, M., Soloveichik, Y., Koshkina, Y., Trubacheva, O., Zlobinskiy, A. [2016] Application of the marine circular electric dipole method in high latitude Arctic regions using drifting ice floes. Journal of Applied Geophysics, 135, 17-31.

Persova, M.G., Soloveichik, Y.G., Vagin, D.V., Koshkina, Y.I. [2016] Grouping of transmitterreceiver positions when using the direct solvers of finite element equation systems in induction logging problems. Proceedings of the 13th International Scientific-Technical Conference on Actual Problems of Electronics Instrument Engineering (APEIE),Novosibirsk, 1, part 2, 305-308.

Persova, M.G., Soloveichik, Y.G., Trigubovich, G.M., Tokareva, M.G. [2013] Methods and algorithms for reconstructing three-dimensional distributions of electric conductivity and polarization in the medium by finite-element 3D modeling using the data of electromagnetic sounding. Izvestiya, Physics of the Solid Earth, 49(3), 329-343. 